UNE CONCEPTION DE MORALITÉ POUR LA THÉORIE ÉCONOMIQUE

Helena Lopes

Fevereiro 2003

WP no 2003/29

DOCUMENTO DE TRABALHO

WORKI NG PAPER 
D I N Â M I A centro de estudos sobre a mudança socioeconómica

\title{
Une Conception de Moralité pour la Théorie Économique *
}

Table des Matiéres

\author{
Helena Lopes
}

WP no 2003/29

Fevereiro de 2003

1. REVENIR, UNE FOIS ENCORE, SUR LA NOTION DE RATIONALITE

2

2. LA PHILOSOPHIE MORALE : UN CHAMP DIVISE

2.1. Présentation générale 5

2.2. Critique de l'utilitarisme 6

2.3. Pour une prise en compte des deux principes 8

3. Moralite et ethique 9

3.1. Moralité et éthique - Distinctions 9

3.2. Fondements de la morale et de l'éthique $\mathbf{1 1}$

4. Normes et VALEURS - LE NORMATIF ET L'AXIOLOGIQUe 12

4.1. Définitions 12

4.2. Normes, valeurs et rationalité économique 15

5. LA CONCEPTION DE LA MORALE CHEZ HABERMAS ET RICOEUR 16

5.1. Habermas et la recherche d'une morale universaliste $\quad \mathbf{1 7}$

5.2. Ricoeur: l'éthique, la morale et la sagesse pratique $\quad \mathbf{2 1}$

6. UNE CONCEPTION DE LA MORALITE POUR L'ECONOMIE

REFERENCES BIBLIOGRAPHIQUES $\quad 29$

\footnotetext{
* Ce travail s'inscrit dans le cadre du projet NORMEC - The Normative Dimensions of Action and Order : The Economics of an Inclusive Europe, financé par le 5ème Programme-Cadre de Recherche et Développement de l’Union Européenne.
} 


\section{Une Conception de Moralité pour la Théorie Économique}

Le désir intime de tout économiste pourrait certainement s'exprimer par cette phrase de Rousseau, en exergue de son Contrat Social : "Je tâcherai d'allier toujours, dans cette recherche, ce que le droit permet avec ce que l'intérêt prescrit, afin que la justice et l'utilité ne se trouvent point divisées”. Pourtant, la science économique contemporaine est, à juste titre, accusée de veiller beaucoup plus aux intérêts qu'à la justice malgré les croissants et louables efforts de beaucoup d'économistes pour rapprocher et même réconcilier la recherche de l'intérêt et les exigences de l'éthique ${ }^{1}$. Mais le domaine de l'éthique ne se prête pas aux contraintes d'une science universaliste. Ainsi, les économistes sont-ils déchirés, au moins depuis la fin du 19ème siècle, entre leur désir intime et le désir de faire science ${ }^{2}$.

L'évacuation de la dimension morale en théorie économique conduit à des impasses, tant lorsque l'économie se veut positive que lorsqu'elle s'assume comme normative, déjà maintes fois dénoncées. Le théoricien a de plus en plus de mal à rendre compte de la coopération et de l'action collective, que ce soit dans la théorie de l'entreprise et la relation de travail ou sur les marchés de produits et les marchés financiers ${ }^{3}$. Les comportements qui sont motivés par des critères de justice ou d'autres valeurs morales plus que par la recherche de l'intérêt personnel - comme la contribution volontaire aux biens publics, le respect de contrats incomplètement spécifiés, et, en général, les conditions de base de l'ordre social - sont inexplicables au vu de la rationalité instrumentale.

L'objectif de ce texte est d'analyser les rapports entre la moralité et la rationalité économique, rationalité instrumentale. L’utilitarisme, principal représentant du libéralisme téléologique ou conséquentialiste, constitue le fondement philosophique de la science économique, laquelle est ainsi fondamentalement étrangère à l'autre grande famille de doctrines morales, la déontologie. L'exigence de rationalité, qui implique que l'on se conduise en toutes circonstances de façon maximisatrice, semble inconciliable avec la prise en compte d'éléments déontologiques tels que des interdits et des obligations absolues.

Partant du postulat que l'on ne saurait se satisfaire de modèles de comportements qui n’intègrent que l'aspect conséquentialiste, notre objectif est triple:

- montrer que la prise en compte d'éléments déontologiques est irréductiblement incompatible avec la formalisation de la rationalité comme maximisation d'une fonction d'utilité;

\footnotetext{
${ }^{1}$ Cf article à venir

${ }^{2}$ Pour reprendre le titre d'un article de Frédéric Lordon

${ }^{3}$ Voir, entre autres, le rapide survol da la littérature in Eymard-Duvernay (2002), Ben-Ner and Putterman (1998), et, en général, les travaux de Sen.
} 


\section{Une Conception de Moralité pour la Théorie Économique}

- montrer que les contraintes morales ne peuvent être réduites au statut d'instrument pour l'atteinte d'une certaine fin;

- ne pas se limiter, toutefois, à enregistrer l'impossibilité de conciliation entre éthique déontologique et rationalité conséquentialiste mais chercher les grandes voies d'une cohabitation possible.

Pour ce faire, nous explorerons certains domaines de la philosophie morale avec l'objectif strict d'y rechercher des contributions qui nous aident à préciser le statut de la moralité dans la conception du comportement économique. Mais tout d’abord, un retour sur la notion de rationalité s’avère nécessaire pour préciser nos objectifs et orienter notre réflexion.

\section{REVENIR, UNE FOIS ENCORE, SUR LA NOTION DE RATIONALITE}

La discussion menée dans cet article concerne exclusivement les rapports de la moralité et de la rationalité telle que définie par la théorie du choix rationnel. Cette notion de rationalité, selon laquelle l'agent peut classer toutes les combinaisons d'action imaginables, ce classement ayant une propriété de cohérence interne dite "transitivité", est constitutive du paradigme dominant en économie, la théorie néo-classique Afin de mieux situer nos objectifs, nous nous inspirerons, dans cette section de la distinction proposée par Demeulenaere (1998) entre trois aspects de cette "rationalité économique".

Selon un premier aspect, que l'on peut qualifier de stratégique, la rationalité concerne la relation entre les moyens et les fins. Demeulenaere (1998, p.53) note pertinemment que n'importe quel type de fin peut donner naissance à une action de ce type, les fins pouvant donc relever ou intégrer des valeurs morales mais les finalités ultimes de l'action échappent au critère de rationalité. L’agent économique peut maximiser son self-interest au sens strict du terme, mais aussi ses intérêts, dans un sens plus large, lesquels peuvent incorporer par exemple la prise en compte des intérêts d'autres personnes. La rationalité instrumentale n’est donc pas nécessairement associée à l'égoisme. Sous cet aspect, la rationalité ne concerne que l’adéquation des moyens.

Certains auteurs, comme Gauthier (1986), ont essayé de démontrer que certaines contraintes morales des éléments déontologiques, donc, - sont instrumentalement rationnelles. Ainsi, par exemple, la coopération serait un bon moyen pour atteindre une fin donnée, égoiste en l’occurrence. 


\section{Une Conception de Moralité pour la Théorie Économique}

Le deuxième aspect de la rationalité concerne les fins elles-mêmes; "les moyens ont encore de l'importance mais l'accent est mis sur la nature des fins, à savoir les intérêts, qui commandent ensuite le choix de certains moyens” (Demeulenaere, 1998, p.52). Cet aspect peut être assimilé, d'une part à la rationalité économique (l'agent cherche à maximiser son intérêt, égoiste et/ou altruiste), d’autre part, à la rationalité conséquentialiste "pour laquelle être rationnel implique que l'on cherche à satisfaire autant que possible l'ensemble des intérêts concernés” (Dupuy, 1996, p.1252). Cette deuxième conception inclut déjà une dimension éthique; c’est celle, par exemple, des auteurs utilitaristes ${ }^{4}$ dont le critère moral ultime est la recherche du bonheur de la communauté désigné comme principe d'utilité. Cet "idéal utilitariste” a été largement abandonné par la théorie économique contemporaine et se trouve confiné à l'économie du bien-être, elle-même soigneusement isolée de l'économie positive.

Dans le cadre de la rationalité économique au sens strict, plusieurs auteurs proposent d'introduire des préférences morales dans les fins poursuivies par les agents, ceux-ci maximiseraient ces préférences au même titre que les préférences d'autre nature. Mais la légitimité de cette intégration d'éléments moraux dans les préférences de l'agent économique est questionnable. Á la fin du 19ème siècle, Pareto, Edgeworth et d'autres ont fondé la science économique justement sur l'opposition entre intérêts et valeurs, entre biens inférieurs et supérieurs, parce-que pour eux, intérêts et valeurs sont des motivations de nature différente. Ainsi, on argumentera que l'intégration d'éléments moraux dans l'analyse soulève la question des motivations de l'agent et des fins poursuivies, question qui ne peut continuer à être éludée par la science économique. Or, cette question est par définition hors du champ de la rationalité économique.

Le troisième aspect de la rationalité, décisif pour la formalisation du choix rationnel, concerne l'ordonnancement total et complet des actions qui permet la constitution d'une fonction d'utilité. Quant à cet aspect, le débat pertinent pour la théorie économique est de savoir si l'intégration de la moralité ou des valeurs n'implique pas de renoncer à l'unidimensionalité des préférences, ou, plus rigoureusement, des dimensions d'évaluation des actions. Les économistes qui veulent rendre compte des comportements qui résultent de la conformité à des normes morales considèrent le sens du devoir, par exemple, comme une préférence de l'agent. Le postulat est que toutes ces préférences, ou plutôt toutes les dimensions d'évaluation des actions, sont passibles d'être pesées les unes contre les autres, c’est-à-dire, sont commensurables. Or, ceci, comme il a été dit ci-dessus, est questionnable.

La majorité des auteurs qui tentent de concilier moralité et rationalité en viennent à introduire deux "niveaux” de délibération ou plusieurs classements de préférences. Il existerait donc des préférences

\footnotetext{
${ }^{4}$ Les termes utilitarisme et utilitariste renvoient, dans ce texte, à la philosophie morale du même nom.
} 


\section{Une Conception de Moralité pour la Théorie Économique}

de différente nature qui interviendraient à des niveaux différents. Mais cette structure de niveaux d'utilité multiples soulève d'importantes difficultés (Caldas, 2001) : Les différents objectifs poursuivis peuvent-ils être agrégés dans une fonction d'utilité? Serait-il suffisant de pondérer plus fortement les préférences de niveau supérieur que celles de niveau inférieur? Ou certains objectifs auraient-ils une priorité absolue, empêchant ainsi l'existence de trade-offs et donc leur incorporation dans une fonction d’utilité unidimensionnelle?

Notre critique porte donc sur les trois aspects de la rationalité : sa nature exclusivement instrumentale, le fait qu'on ne puisse y inclure des fins morales sans mettre en cause la théorie du choix rationnel. Mener à bien cette critique oblige à procéder à des précisions terminologiques et à proposer une conception déterminée de la moralité. Ce sera l’objet des sections suivantes.

\section{LA PHILOSOPHIE MORALE : UN CHAMP DIVISE}

\subsection{Présentation générale}

La question principale de la philosophie morale est celle des voies du bonheur individuel et collectif. On y distingue deux grandes classes de doctrine morales. Les doctrines téléologiques (ou conséquentialistes, parmi lesquelles s'inscrit l'utilitarisme) subordonnent le juste au bien : une action est juste ou morale si elle contribue à la maximisation du bonheur (ou de l'utilité) du plus grand nombre. Les actions sont évaluées en fonction de leurs conséquences. La rationalité instrumentale est la forme de la rationalité propre à ces doctrines éthiques puisque les actes ne sont que des moyens au service d'une fin posée en extériorité ou transcendance par rapport au sujet moral (Dupuy, 1994, p.122).

Les doctrines déontologiques (dont le principal représentant est Kant), par contre, donnent une priorité absolue au juste sur le bien; aucun acte ne peut être légitimé par la raison que ses conséquences contribuent à l'accroissement de l'intérêt général. Une action doit être juste en soi, les normes et principes moraux doivent être respectés pour eux-mêmes.

Ces deux classes de doctrines se sont beaucoup opposées. Pour les déontologistes, le domaine de la morale, régi par l’impératif catégorique (“Agis uniquement d’après la maxime qui fait que tu puisses 


\section{Une Conception de Moralité pour la Théorie Économique}

vouloir qu'elle devienne une loi universelle”), échappe à la rationalité instrumentale qui ne connait que les impératifs hypothétiques (“Si tu veux telle chose, alors fais ceci”). L’approche déontologique privilégie la conformité à certaines normes tandis que les principes téléologiques relèvent d'une évaluation des actions sur la base d'un but à promouvoir. Ainsi, l'éthique déontologique et la rationalité instrumentale semblent irréductiblement opposées ${ }^{5}$, le désir de Rousseau de réconcilier justice et intérêt serait à jamais utopique.

Toutefois, considérer que les deux doctrines s'opposent n'est pas la seule perspective envisageable. Par exemple, certains auteurs défendent la thèse de la priorité du déontologique sur le téléologique (Picavet, 1998, p.68-69). Par ailleurs, l'utilitarisme de la règle admet des principes moraux (comme la valeur absolue de la promesse) dont la justice n'est pas définie à l'aune du principe d'utilité. Le vrai problème est toujours de savoir si le pôle téléologique appelle ou non des méthodes d'analyse différentes de celles qui conviennent à l'éthique déontologique.

Adam Smith, père de l'économie politique, semble vouloir concilier les deux types de principe, ce qui explique la divergence des opinions, entre les spécialistes, sur sa qualification comme auteur utilitariste. En effet, on trouve dans son ouvrage des citations contradictoires : "toutes les institutions de la société ... sont jugées uniquement par le degré avec lequel elles tendent à promouvoir le bonheur de ceux qui vivent sous leur juridiction. C’est là leur seul usage et leur unique but” (Smith, 1999, p.259); mais aussi "la bienfaisance est l'ornement qui embellit et non la fondation qui supporte le bâtiment ..., la justice est le pilier principal qui soutient toute la construction [la société]” (op.cit., p.141).

\subsection{Critique de l'utilitarisme}

Puisque l’utilitarisme est la "philosophie spontanée" des économistes et que l'objectif de ce texte est de persuader le lecteur que la science économique devrait (ré)intégrer non seulement la morale utilitariste mais aussi des éléments de morale déontologique, il nous semble pertinent de mentionner certaines de critiques les plus fréquemment adressées à l’utilitarisme ${ }^{6}$.

Du point de vue philosophique, la critique essentielle semble être le fait que l'utilitarisme aboutit logiquement à légitimer le sacrifice : la poursuite d’une “utilité générale” porte en elle la nécessité du

\footnotetext{
${ }^{5}$ Et, au niveau philosophique le plus abstrait, ils le sont effectivement.

${ }^{6}$ Nous ne prétendons bien évidemment pas rendre compte de tous les débats et polémiques qui vont en se multipliant à propos de l'utilitarisme. Par ailleurs, l'éthique déontologique et son rigorisme sont également, et tout aussi justement, objet de critiques radicales.
} 


\section{Une Conception de Moralité pour la Théorie Économique}

sacrifice de l'individuel au nom du bien général ${ }^{7}$. En effet, par exemple, quelles sont les bases qui permettent, dans le cadre de cette théorie, de condamner la personne qui a assassiné un voisin particulièrement nuisible, si son geste a eu l'effet d'augmenter l'utilité de toutes les personnes du quartier $^{8}$ ? Ce passage de l'individuel au collectif est apparu comme un obstacle à la cohérence du système ${ }^{9}$ dès la genèse de l'utilitarisme et les pères fondateurs ont proposé des solutions très diverses pour essayer de résoudre le problème ${ }^{10}$.

En d'autres termes, si le bonheur de la société est le critère suprême de la morale utilitariste, la liberté individuelle y apparait comme un bien subordonné. Si une liberté particulière contribue au bonheur général, elle est considérée comme bonne, si elle diminue la somme globale des utilités, elle est considérée non souhaitable. Or, cette approche est radicalement différente de l'anthropologie de Kant pour qui la liberté est entendue comme faculté d'arrachement à ses intérêts propres. "Seule une valeur catégorique peut constituer une véritable limite à mes intérêts individuels. Or tout impératif de ce type requiert précisément... une faculté de s'arracher à soi, un concept de liberté qui s'oppose à la rationalité des intérêts” (Canto-Sperber, 1992, p.242-243).

On peut d'ores et déjà percevoir que la position que l'on adopte par rapport à ces débats va fortement influencer les options en matière de théorisation et de modélisation du comportement et de l'ordre (de la régulation) économiques. Sont ici en cause surtout les deuxième et troisième aspect que nous avons distingué ci-dessus à propos de la notion de rationalité.

Une des interprétations possibles de l'utilitarisme est que peu importent les raisons qui meuvent les comportements - bienveillance ou envie, altruisme ou égoisme, amour ou haine -, seuls comptent les effets des actions sur le "niveau de prospérité générale”. Cette interprétation, devenue morale de l'efficience (avec le critère de Pareto), a influencé toute la pensée économique moderne et a ainsi nourri l'illusion d'une science axiologiquement neutre, décisivement coupée de toute conception éthique $^{11}$. Le "monde analytique" de la théorie économique ne présuppose aucune conception

\footnotetext{
${ }^{7}$ Pour un développement clair et systématique de cette critique et des réponses (partielles) conséquentialistes à cette critique , voir Dupuy (1996)

${ }^{8}$ Bien sur, tous les auteurs conséquentialistes proposent des solutions à ces objections, lesquelles passent toujours par l'imposition de contraintes neutres, sous la forme, par exemple, d'institutions, lois ou autres.

${ }^{9} \mathrm{cf}$ citations de Smith et Hume in Dupuy, 1992, p.113.

${ }^{10}$ Ces solutions ont été systématisées par Élie Halévy (1995 : “La formation du radicalisme philosophique”, PUF) comme suit : identification spontanée de l'intérêt privé à l'intérêt général grâce à la bienveillance ou à la sympathie (la fusion des intérêts) et automatisme naturel de la main invisible (automatisme qui concourt vers une identité naturelle des intérêts) chez Smith; intervention du législateur chez Bentham (législateur dont la fonction est l'identification artificielle des intérêts).

${ }^{11}$ D’où le succès des travaux de Gauthier auprès de la communauté des économistes.
} 


\section{Une Conception de Moralité pour la Théorie Économique}

particulière du "bien”, ni l'existence de “devoirs moraux"; la société est, dans ce cadre, un simple mécanisme que tous utilisent en commun pour atteindre des fins individuelles.

\subsection{Pour une prise en compte des deux principes}

On ne peut que prendre acte du fait que rationalité instrumentale et déontologie constituent deux formes d'intuitions morales qu'il est impossible de réunir en un seul système éthique cohérent. Mais, si ces deux formes existent parmi les êtres humains, la prise en compte d'une seule d'entre elles dans une science qui se veut descriptive est-elle légitime? Est-elle possible et souhaitable? Ne prétend-on pas ainsi évacuer quelque dimension de la condition humaine jugée moins confortable? Ricoeur se prononce : “le souhait de vivre bien... précède... l'interdiction sous les traits de laquele le sujet moral rencontre l'obligation. Seule, selon moi, l'irruption de la violence dans les rapports humains impose de passer du style téléologique de l'éthique au style déontologique de la morale, en bref, d'Aristote à Kant” (Ricoeur, 1995, p.111).

Selon Ricoeur, donc, il est impossible, dans la "raison pratique”, qu'est la raison économique, de ne prendre en considération qu'un des critères éthiques. C'est là une attitude que nous souscrivons. Et Durkheim (1996) nous fournit également une perspective particulièrement intéressante des rapports entre la notion du bien et celle du devoir selon laquelle ces deux principes ne peuvent être isolés l'un de l'autre mais, au contraire, coexistent : "Nous éprouvons un plaisir sui generis à faire notre devoir. La notion de bien pénètre jusque dans la notion de devoir comme la notion de devoir et d'obligation pénètre dans celle de bien” (op. cit., p. 63). Selon cet auteur, les fins morales sont, entre autres, objets de désirs, ce qui va contre l’hypothèse kantienne selon laquelle il existerait hétérogénéité entre la Raison et la Sensibilité. Durkheim refuse catégoriquement d'admettre un ordre de priorité même logique entre l’idée de bien et celle de devoir : “Jamais du désirable on ne pourra tirer l'obligation, puisque le caractère spécifique de l'obligation est de faire, en quelque mesure, violence au désir. Il est aussi impossible de dériver le devoir du bien (ou inversement) que de déduire l'altruisme de l'égoisme" (op. cit., p.65) ${ }^{12}$.

Et nous achèverons cette section par une citation qui nous semble s'adresser particulièrement aux économistes : “On échappe ainsi et à l'empirisme utilitaire qui tente bien d'expliquer rationnellement la morale, mais en niant ses caractères spécifiques, en ravalant ses notions essentielles au même rang que les notions fondamentales des techniques économiques, et à l'apriorisme kantien qui... décrit plus qu'il n'explique... C'est que ces manières de voir, qui s'opposent chez les moralistes, ne s'excluent

\footnotetext{
${ }^{12}$ Durkheim déjouait ainsi, avant la lettre, les tentatives de fonder la moralité sur la rationalité instrumentale, comme le fit Gauthier près d'un siècle plus tard.
} 


\section{Une Conception de Moralité pour la Théorie Économique}

que dans l'abstrait ... elles ne font qu'exprimer des aspects différents d'une réalité complexe” (op. cit., pp.82-83).

\section{MORALITE ET ETHIQUE}

\subsection{Moralité et éthique - Distinctions}

Notre objectif dans cette section est de mieux comprendre ce que recouvrent les termes de moralité et d'éthique, termes que l'on trouve souvent, mais rarement définis, dans la littérature économique. Il nous est rapidement apparu que les définitions sont multiples et quelquefois contradictoires ${ }^{13}$.

Il nous faut tout d'abord préciser que nous utiliserons de façon indifférenciée les termes "morale” et "moralité", auxquels nous semblent correspondre “morals” et “morality” dans la littérature anglosaxonne.

D’éthymologie grecque - ethos -, le mot éthique est pour certains auteurs entendu comme synonyme du mot morale - formé sur le latin mores - mais, considérant la distinction intéressante puisque l'usage les a effectivement différenciés, nous chercherons plutôt à établir une distinction entre les deux termes. Généralement, la moralité est considérée comme un sous-domaine de l'éthique et est associée aux systèmes qui, à la suite de Kant, basent la morale sur les notions de devoir et d'obligations, l'éthique étant réservée à la perspective plus aristotélicienne de raison pratique basée sur la notion de vertu, laquelle évite la séparation des considérations morales et des considérations pratiques (Blackburn, 1996).

Dans son sens le plus large, l'éthique désigne le questionnement sur le bien et le mal (et sur le juste) et cherche à déterminer quelles conduites sont moralement bonnes et comment déterminer la validité des jugements de valeur. L'éthique s'occupe, entre autres, des normes et des valeurs qui régissent ou doivent régir les relations de chaque individu aux autres. Les normes éthiques (à la différence des normes légales) ne sont pas imposées de façon contraignante mais font au contraire appel à un sentiment de responsabilité face à une entité autre que soi - Dieu, la société, l’humanité, le spectateur impartial. “L'attitude éthique consiste pour l'agent à se soumettre volontairement à la transcendance et

\footnotetext{
${ }^{13}$ Encore une fois, nous ne prétendons pas rendre compte de la littérature existante en ce domaine, tâche pour nous tout à fait hors d'atteinte.
} 
à l'universalité d'une règle qu'il a lui-même posée en se la donnant à lui-même” (Dupuy, 1994, p.122).

De son côté, la moralité est "tout ce qui, dans l'ordre du bien et du mal, se rapporte à des lois, des normes, des impératifs” (Ricoeur, 1993, p. 62). Parmi les multiples caractérisations du "moral”, nous avons retenu celles de Skorupski (1998) et de Durkheim (1996, pp. 51-54).

Selon Skorupski, la moralité peut être caractérisée par :

1. sa fonction : les règles et convictions morales ont pour fonction de promouvoir l'épanouissement de l'homme et de permettre une vie sociale marquée par la coopération (... reste à savoir si cette coopération n’est pas plus une conséquence qu’une fonction de la moralité);

2. sa suprématie par rapport à d'autres délibérations : les obligations morales sont telles qu'elles doivent être respectées quelles que soient les circonstances;

3. les sentiments spécifiques associés aux évaluations morales : une obligation est morale si et seulement si des sentiments comme le blâme envers un individu qui ne respecte pas l'obligation sont justifiés.

La perspective caractérisée en 1. reproduit la conception de beaucoup de conséquentialistes d'aujourd'hui et adoptée par beaucoup d'économistes : la morale a un caractère instrumental -tout comme la rationalité-, les normes/préférences/dispositions morales servent à/pour maximiser l'utilité individuelle et/ou collective en rendant possible la coopération (dans les cas de dilemmes moraux comme le dilemme du prisionnier et la provision de biens collectifs, par exemple). L'aspect 2. s'inscrit dans une perspective plus kantienne et rappelle l'impératif catégorique; rien n’est dit, toutefois, sur la "nature” de la moralité. En revanche, l'aspect 3. se démarque de Kant et rejoint les philosophes des Lumières Écossaises, comme Smith et Hume, qui analysaient la moralité en termes de sentiments moraux.

Durkheim caractérise le fait moral par 1. son caractère d'obligation; par 2. une certaine désirabilité (rompant, lui aussi, avec Kant) et aussi par 3. le fait que la morale est nécessairement associée à un collectif d'individus (“Jamais la qualification de moral n’a été appliquée à un acte qui n’a pour objet que l’intérêt de l'individu”).

\subsection{Fondements de la morale et de l'éthique}

Beaucoup de philosophes ont cherché à fonder universellement la moralité et l'éthique (à l'exception des “sceptiques moraux” pour qui, précisément, les normes et les valeurs ne peuvent pas être fondés) 


\section{Une Conception de Moralité pour la Théorie Économique}

de façon à valider les principes éthiques avancés. Á première vue, la tâche est difficile, sinon hors d'atteinte, puisqu'il n'existe pas d'accord universel sur ce qu'est le bien. Deux solutions, entre autres, sont apparues qui ont été largement reprises ou souscrites par les économistes : la solution utilitariste et la solution "procédurale" qui essaie de démontrer que les normes et valeurs résultent de principes procéduraux (et non substantiels) irrécusables.

Pour les utilitaristes, il existe de fait un bien reconnu comme tel par tous : le bonheur ou l'utilité. Ce bien est donc un critère universel pour mesurer la justice et l'injustice des actions humaines. Malgré son indubitable pertinence, l'utilitarisme a ses limites, dont certaines ont été rapidemment mentionnées à la section précédente, et nous ne les reprendrons pas ici.

L'autre solution est celle qui tente de fonder l'éthique en raison, une raison hors du temps et de l'histoire. Pour certains, ce projet, qui est celui des Lumières françaises, aurait définitivement échoué, et la raison instrumentale, déjà réduite à n'être plus qu'une rationalité en fonction d'une fin, a désormais abandonné jusqu’à la fixation même de cette fin : "La raison est calculatrice. Elle peut établir des vérités de fait et des relations mathématiques, mais rien de plus. Dans le domaine de la pratique, elle ne peut parler que des moyens. A propos des fins, elle doit se taire" (citation de Horkheimer in Habermas, 1986, p.63).

Avant de continuer, il est important de remarquer que cette critique s’applique particulièrement bien à la rationalité économique qui s’est de tout temps évertuer à écarter les considérations axiologiques hors de son champ.

Mais cette affirmation de Horkheimer est contestée, et le projet des Lumières est repris, depuis Kant, par certaines éthiques cognitivistes (parmi lesquelles celles de Habermas et de Rawls) qui s'attèlent toutes au même dessein : analyser les conditions qui rendraient possibles une évaluation impartiale. S’il n’y a pas possibilité de consensus sur le bien, il y a possibilité de consensus sur les procédures à suivre pour définir le bien. Là encore, il s'agit d'élaborer une conception de la moralité sans se référer à des valeurs.

En guise de conclusion sur l'éthique et la moralité, notre proposition est de garder, à des fins d'analyse économique, la notion de moralité. En effet, d’une part, l'éthique nous semble relever d'une réflexion trop fortement philosophique et générale sur le bien et le mal pour pouvoir être importée en économie sans prêter à confusion. D’autre part, comme déjá mentionné, ce qui nous semble absent de la théorisation économique, ce sont surtout les éléments d’une éthique déontologique, désignés 


\section{Une Conception de Moralité pour la Théorie Économique}

précisément par le terme de moralité. Car, bien que plus ou moins abandonnée, l'éthique utilitariste est supposée être au fondement de la science économique, il s’agit donc de la faire cohabiter avec des éléments de moralité, c’est-à-dire des devoirs et des obligations.

\section{NORMES ET VALEURS - LE NORMATIF ET L’AXIOLOGIQUE}

\subsection{Définitions}

Tout comme dans le cas de l'éthique et de la morale, on trouve dans la littérature philosophique des positions très différentes quant à la définition et aux rapports entre ces deux notions. Certains auteurs ignorent la distinction entre normes et valeurs, d'autres proposent la réduction des unes aux autres, d'autres encore s'attèlent à établir des distinctions fines et des différences quant au champ d'application. Dans ce qui suit, nous nous inspirerons en particulier de l'article de Ogien (1992).

Ogien commence par souligner que normes et valeurs semblent appartenir à des familles différentes : les théories des normes traitent de règles, principes, devoirs tandis que les théories des valeurs traitent plutôt de bien et de mal. Mais ces deux notions appartiennent à la même famille : celle des notions prescriptives par opposition aux notions -exclusivement- descriptives, même si les normes représentent des énoncés prescriptifs ou directifs (obligation, interdiction, permission) alors que les valeurs s’énoncent en termes évaluatifs (appréciation en termes de bien, mal, etc.).

Les normes sont généralement définies comme des règles de conduite - dans le champ de la morale, les règles sont usuellement appelées normes - qui spécifient le comportement approprié dans certaines circonstances. On y trouve donc un aspect de régularité comportementale et un aspect de prescription comportementale. Ogien propose de qualifier ces énoncés moraux de normatifs, ces énoncés portant sur ce qu'il faut faire et s'appliquant à des actions humaines (référence à une action, à un certain type d’agent et à des circonstances déterminées).

La norme ne se résume pas au prescriptif; elle intègre également un caractère appréciatif (la norme, c'est ce qu'il est bien ou correct de faire) et un caractère descriptif (comme il est patent dans la définition donnée plus haut). Il est important d'insister sur ces trois aspects des normes, qui lui confèrent chacun des contenus distincts Au sens prescriptif et appréciatif, la norme semble impliquer l'existence de sanctions positives ou négatives, formelles ou informelles.. L’aspect appréciatif met en 


\section{Une Conception de Moralité pour la Théorie Économique}

évidence que la norme n’est pas seulement ce qui s'impose à nous, c'est aussi ce qui est apprécié, subjectivement désiré ou jugé légitime (cf réflexions de Durkheim citées plus haut); c’est justement cet aspect qui distingue les normes des règles, lois et règlements dont la violation est, elle aussi, passable de sanctions. L'analyse des normes impose ainsi de tenir compte de l'aspect public, objectif, contraignant de celles-ci.

D’après Ricoeur (1993, p.64), le terme de valeur se situe entre l'éthique et la morale. Il correspond à ce que les anciens nommaient vertus et recouvre des entités telles que la justice, l'égalité, la tempérance, l'amitié, etc. Si les normes étaient des énoncés moraux prescriptifs, les valeurs sont des énoncés évaluatifs - dans le mot valeur, il y a le verbe évaluer, qui renvoie à préférer -, souvent désignés d'axiologiques. De même, si les normes ont par définition un caractère public, objectif, il existe beaucoup de définitions purement subjectives de la valeur, lesquelles s’inscrivent dans la lignée de Hume pour qui la valeur d'un objet ou d'une action était une fonction des intérêts ou des émotions de l'agent. Plus récemment, des théories objectives de la valeur sont apparues qui insistent plutôt sur la stabilité des valeurs et leur indépendance vis-à-vis des - mais non l'absence de relation avec lesintérêts et émotions (Ogien, op. cit., p.1055).

L’approche subjective des valeurs est critiquée parce-qu'elle ne distingue pas entre ce qui est désiré et ce qui est désirable/valable, entre les désirs et les valeurs. L’origine de la confusion entre le désiré et le valable est à rechercher auprès des utilitaristes pour qui ce qui est désirable n'est rien d'autre que ce que l'on désire. Or, ce qui est désirable, valable n’est pas ce qu'on désire mais ce que l'on devrait désirer (rappelons que les valeurs appartiennent à la famille des notions prescriptives).

Pour Ricoeur (1993), évaluer et préférer est l'apanage d’un être de volonté et de liberté, l'évaluation marque ainsi l'autonomie de la personne humaine. Par ailleurs, les valeurs se présentent comme des étalons de mesure, qui transcendent, donc, les évaluations individuelles. Les valeurs ne sont pas, toutefois, des essences éternelles mais sont au contraire liées à une histoire des moeurs, à une culture. La notion de valeur est donc une notion de compromis entre le désir de liberté et les situations déjà éthiquement qualifiés, entre la socialisation et le jugement moral privé. C’est cette référence à l'évaluation par autrui qui élève le valable au-dessus du simple désirable.

Nous proposons ainsi que l'on retienne la notion de valeur, telle que définie par Ricoeur, comme pertinente pour la théorie économique dans la mesure où elle permet d'établir une distinction entre valeurs et désirs.

\subsection{Normes, valeurs et rationalité économique}




\section{Une Conception de Moralité pour la Théorie Économique}

S'il est important de distinguer normes et valeurs, on ne peut opposer les valeurs - relatives à l'idéal, à la liberté, au subjectif - aux normes -relatives à l'impératif, à l'objectif, au contraignant. Les deux notions, bien que distinctes, s'enchevêtrent, et, en ce qui concerne l'analyse économique, elles nous paraissent y avoir chacune son domaine et son intérêt, étant irréductibles l'une à l'autre. Ces notions sont essentielles pour l'explication, pour la compréhension et aussi pour la prédiction des comportements économiques. Les normes apparaissent comme des solutions plus ou moins routinisées aux problèmes de coopération et d'action collective, ces normes s'inspirant de valeurs partagées plus générales. La notion de valeur, à son tour, permet de diminuer l'importance de l'idée de sanction dans l'explication de l'action et de faire place à l'autonomie de l'agent. C'est pourquoi, entre autres, il est insatisfaisant de modéliser les valeurs sous forme de contraintes à la maximisation de l'utilité. Si les normes peuvent être -en partie seulement, on y reviendra- analysées en termes de rationalité instrumentale, il n'en est pas de même pour les valeurs.

En adoptant exclusivement une théorie subjectiviste de la valeur, la science économique actuelle s'inscrit dans la mouvance de l'orthodoxie philosophique anglo-saxonne ${ }^{14}$. Les théories utilitaristes de la valeur ont évolué de l'hédonisme benthamien vers une notion d'utilité à la Becker, laquelle intégre de façon indiscriminée intérêts et devoirs, désirs et valeurs. Au passage, la notion d'utilité a été “désubstantialisée”, comme il est patent dans la théorie des préférences révélées. L’utilité, seule valeur économique, est subjective, la fonction d'utilité est à valeur unique.

Pourtant, au sein même de la philosophie analytique anglo-saxonne, les tenants des théories objectivistes de la valeur réclament aujourd'hui une plus grande indépendance entre les valeurs et les intérêts tandis que les rationalistes neo-kantiens affirment que la raison pratique doit indiquer non seulement ce qui est instrumentalement valable (valable pour l'atteinte de fins déterminées ) mais aussi quelles sont les fins qui sont dignes de valeur (Thomas, 1998, p.582). Une autre fonction devrait ainsi être assignée à la rationalité économique, celle de discuter des fins. Reste à savoir si cette fonction peut être menée à bien dans le cadre actuel du choix rationnel.

Certains auteurs soulignent le fait qu'on ne peut opposer de façon simpliste intérêts et valeurs. En effet, les intérêts sont eux-mêmes l'objet d'évaluations. De même, on ne peut opposer fins et moyens en réservant les valeurs à la seule évaluation des fins. Soit. Il reste que valeurs et intérêts sont, comme nous avons essayé de le démontrer dans cette section, irréductibles l'un à l'autre mais, aussi et surtout,

\footnotetext{
${ }^{14}$ laquelle est elle-même fortement influencée par la théorie du choix rationnel.
} 


\section{Une Conception de Moralité pour la Théorie Économique}

incommensurables : ils ne peuvent être pesés les uns contre les autres sur un même plan; ils participent de dimensions différentes.

L'objectif des rationalistes est de trouver des critères moraux universels. Or, on l'aura remarqué, la définition de valeurs donnée ci-dessus comporte une dimension axiologique et donc, historique et culturelle. Peut-on donc, en économie, garder la notion de valeurs sans renoncer à faire une science pure? La réponse, bien évidemment, est non. Si l'économie est une science morale (Caldas, 2002), dans le sens où elle est, d'une part, forcément empreinte d'éléments axiologiques et où elle exerce elle-même, d'autre part, une influence sur les valeurs en vigueur dans une société/civilisation donnée, elle doit abandonner la prétention universaliste et assumer sa normativité.

\section{LA CONCEPTION DE LA MORALE CHEZ HABERMAS ET RICOEUR}

C'est à partir de l'oeuvre de ces deux philosophes, dont la pensée intègre l'essentiel de la réflexion éthique et morale jusqu'à nos jours, que l'on précisera notre proposition de conception de la moralité pour l'économie. La différence très substantielle de leurs conceptions nous fournira une idée de la diversité des positions possibles sur ce sujet.

\subsection{Habermas et la recherche d’une morale universaliste}

Le projet de Habermas est de fonder l'éthique en raison en adoptant la forme d'une logique de l'argumentation/discussion. Il s'inscrit ainsi dans le courant rationaliste ${ }^{15}$ neo-kantien (et non dans le courant herméneutique, comme Ricoeur) qui essaie de donner aux valeurs un fondement rationnel, c'est-à-dire, de détacher les normes de la raison de tout contexte particulier pour en faire un invariant normatif universel. Habermas tente ainsi et d'échapper au positivisme et d'échaper à l'historicisme.

Son point de départ est, tout comme celui des herméneutiques, de faire du langage tout à la fois le principe de raison et la raison en acte. Selon lui, la raison est cooriginaire au langage. Sa conception de

\footnotetext{
${ }^{15} \mathrm{Et}$, plus particulièrement, cognitiviste.
} 


\section{Une Conception de Moralité pour la Théorie Économique}

la morale s’appuie sur la théorie psychologique de la morale de Kohlberg (laquelle dérive largement de Piaget) selon laquelle logique et morale se développent, l'une et l'autre, en une succession de stades, chacun des stades étant considéré formellement comme une structure plus équilibrée que le précédent (Habermas, 1986, p. 56). Ainsi, il peut y avoir universalisme moral parce-qu’il y a un universalisme cognitif du développement moral.

Le principe d'une éthique de la discussion se réfère à une procédure qui consiste à honorer par la discussion des exigences normatives de validité. L'éthique de la discussion est donc formelle; elle ne livre pas d'orientations relatives au contenu mais une manière de procéder : la discussion pratique. L’objet de cette procédure n'est pas de produire des normes légitimées mais de tester la validité des normes proposées. Les discussions pratiques portent sur un contenu qu'elles reçoivent de l'extérieur; les participants doivent régler par le consensus les problèmes ${ }^{16}$ et conflits sociaux qui sont les leurs. Les points de vue axiologiques qui n’aboutissent pas à un consensus seront abandonnés. Cette procédure est donc formelle mais non au sens où il y a abstraction des contenus (op. cit., p.125). Pour Habermas, le domaine de validité de l'éthique déontologique doit se limiter aux questions susceptibles d'être débattues rationnellement et d'aboutir au consensus.

La fondation en raison de l'éthique de Habermas comporte deux temps : le premier consiste à introduire un principe d'universalisation " $U$ " comme règle d'argumentation pour les discussions pratiques; le second consiste à démontrer que "U" a valeur universelle, ie, qu'il outrepasse la perspective d'une culture déterminée (p.131). Il modifie donc l'impératif kantien “au lieu d’imposer à tous les autres une maxime dont je veux qu'elle soit une loi universelle, je dois soumettre ma maxime à tous les autres afin d'examiner par la discussion sa prétention à l'universalité" (p.88). La différence est d'ampleur puisqu'elle signifie que la fondation en raison des normes et commandements ne peut se faire de manière monologique (au moyen d'une argumentation hypothétiquement développée en pensée) mais requiert la discussion ${ }^{17}$. C'est dans la sphère de l'intercommunication et non plus de la conscience réputée solitaire que la fondation dernière de la moralité est à rechercher. "Le principe d'universalisation fonctionne comme un couteau qui tranche entre "le bien” et "le juste", entre les énoncés évaluatifs et les énoncés strictement normatifs” (p. 125). Les valeurs culturelles, bien que porteuses d'une exigence de validité intersubjective, ne peuvent prétendre d'emblée à une validité normative au sens strict; elles peuvent tout au plus se porter candidates au statut de normes susceptibles de promouvoir un intérêt universel.

\footnotetext{
${ }^{16}$ Habermas parle de “questions pratiques” qu’il définit comme des questions qui ont trait à la “vie bonne”.
} 


\section{Une Conception de Moralité pour la Théorie Économique}

Ainsi, pour Habermas, il y a distinction nette entre normes et valeurs : les normes peuvent être soumises aux exigences d'une justification strictement morale tandis que les valeurs englobent les orientations axiologiques inhérentes aux modes de vie individuels et collectifs. "Autrement dit, les questions morales peuvent être tranchées rationnellement, du point de vue de l'universalisation des intérêts ou du point de vue de la justice et elles se différencient des questions d'evaluation qui ... se présentent comme des questions relatives à la "vie bonne”"(p. 129-130). Ainsi, le jugement moral se libère, chez Habermas, des conventions locales et de la tonalité historique d'une forme de vie particulière. Toutefois, reconnait-il, toute morale universaliste doit être complétée par une moralité sociale (une morale sociale concrète), la seule qui fournisse des motivations pour l'action, dans le domaine de la raison pratique.

Pour simplifier et pour situer les positions de Habermas par rapport aux notions que nous avons présentées ci-dessus, on pourrait dire que l'éthique de Habermas définit une morale universaliste (constituée des normes d'action prescriptives et qui ont valeur déontologique) qu'il distingue de la moralité sociale, laquelle intégre les valeur, non-moralisables et non-formalisables.

Également intéressante pour notre propos est la typologie des actions de Habermas (Munck, ..., pp. 159-160):

- Le premier type d'action, dit téléologique, a pour objectif la réalisation de plans déterminés par des moyens appropriés - c’est l'activité instrumentale. La finalité de l’action est déterminée a) indépendamment des moyens mis en jeu, et b) comme un état qu’il s’agit de produire causalement. Lorsque l'activité téléologique inclut dans son champ non seulement la nature, mais d'autres agents, elle devient stratégique (l'exemple type est la théorie des jeux). Dans cette activité, les agents s'orientent exclusivement vers le succès, c'est-à-dire qu'ils se concentrent exclusivement sur les conséquences de leur action. Le degré de coopération et de stabilité résulte de ce qui détermine les intérêts des participants, sans recours aux normes des agents ni au langage. Les mécanismes de coordination sont la monnaie et le pouvoir.

- L'activité communicationnelle, par contre, se produit lorsque les agents acceptent d'accorder leurs projets d'action et de ne tendre vers leus buts respectifs que si une entente est atteinte. Dans cette activité, a) il est impossible de définir les fins indépendamment des moyens (la parole), b) l'entente ne peut être obtenue par une action causale mais doit passer par la compréhension partagée. Dans ce cas, le mécanisme de coordination de l'action est l'intercompréhension.

\footnotetext{
${ }^{17}$ Avec l'”Autre” dirait Ricoeur. Cette idée de l'incomplétude de la Raison “personnelle” nous parait particulièrement intéressante.
} 
L’activité communicationnelle, en plus de la dimension téléologique qu'elle présuppose également (il s'agit toujours d'atteindre des buts), dégage la dimension communicationnelle qui se manifeste dans l’interprétation commune de la situation (Habermas, 1986, p.149).

Pour fonder en raison les stades moraux selon une logique du développement, Habermas établit un lien entre les perspectives sociales de Kohlberg et ses propres stades d'interaction, dont nous reproduisons ci-dessous une systématisation simplifiée (pp. 180-181).

\section{Les stades d'interaction, les perspectives sociales et les stades moraux ${ }^{18}$}

\begin{tabular}{|c|c|c|c|c|c|}
\hline $\begin{array}{c}\text { Types } \\
\text { d'action/ } \\
\text { Struct. } \\
\text { cognitives }\end{array}$ & $\begin{array}{l}\text { Concept } \\
\text { d'autorité }\end{array}$ & $\begin{array}{l}\text { Concept de } \\
\text { motivation }\end{array}$ & $\begin{array}{l}\text { Perspectiv } \\
\text { e sociale }\end{array}$ & $\begin{array}{c}\text { Représentation } \\
\text { de la justice }\end{array}$ & $\begin{array}{c}\text { Stades } \\
\text { jugeme } \\
\text { nt } \\
\text { moral }\end{array}$ \\
\hline $\begin{array}{l}\text { Interaction } \\
\text { guidée par } \\
\text { l'autorité }\end{array}$ & $\begin{array}{l}\text { Autorité des } \\
\text { personnes de } \\
\text { référence }\end{array}$ & $\begin{array}{l}\text { Orientation } \\
\text { en fonction } \\
\text { récompense/ } \\
\text { punition }\end{array}$ & $\begin{array}{c}\text { Perspective } \\
\text { égocentriqu } \\
\text { e }\end{array}$ & $\begin{array}{l}\text { Complémentarit } \\
\text { é de l'ordre et } \\
\text { de l'obéissance }\end{array}$ & 1 \\
\hline $\begin{array}{l}\text { Coopération } \\
\text { guidée par } \\
\text { des intérêts }\end{array}$ & idem & idem & idem & $\begin{array}{l}\text { symétrie des } \\
\text { compensations }\end{array}$ & 2 \\
\hline $\begin{array}{l}\text { Activité de } \\
\text { rôle }\end{array}$ & $\begin{array}{c}\text { Autorité } \\
\text { intériorisée d'un } \\
\text { aribtraire supra- } \\
\text { individuel } \\
\end{array}$ & $\begin{array}{l}\text { Obligation } \\
\text { contre } \\
\text { inclination }\end{array}$ & $\begin{array}{l}\text { Perspective } \\
\text { du groupe } \\
\text { original }\end{array}$ & $\begin{array}{l}\text { Conformité au } \\
\text { rôle }\end{array}$ & 3 \\
\hline $\begin{array}{l}\text { Interaction } \\
\text { régie par des } \\
\text { normes }\end{array}$ & $\begin{array}{c}\text { Autorité } \\
\text { intériorisée de la } \\
\text { volonté imperson. } \\
\text { collective - } \\
\text { légitimité } \\
\end{array}$ & Idem & $\begin{array}{l}\text { Perspective } \\
\text { d'un } \\
\text { collectif }\end{array}$ & $\begin{array}{l}\text { Conformité au } \\
\text { système } \\
\text { normatif } \\
\text { existant }\end{array}$ & 4 \\
\hline Discussion & $\begin{array}{l}\text { Valeur idéale } \\
\text { contre valeur } \\
\text { sociale }\end{array}$ & $\begin{array}{l}\text { Autonomie } \\
\text { contre } \\
\text { hétéronomie }\end{array}$ & $\begin{array}{l}\text { Perspective } \\
\text { adoptée en } \\
\text { fonction de } \\
\text { principes }\end{array}$ & $\begin{array}{l}\text { Orientation en } \\
\text { fonction des } \\
\text { principes de } \\
\text { justice }\end{array}$ & 5 \\
\hline
\end{tabular}

Devant ce tableau, on ne peut s'empêcher de remarquer que l'homo oeconomicus parait s'être arrêté au stade 2 du développement moral, dans le meilleur des cas. Le champ des décisions économiques se restreint-il aux deux premiers types d'interaction (comme l'ont établi Edgeworth et d'autres marginalistes)? N'est-il pas de problèmes économiques qui n'exigent l'intercompréhension et la communication (nous pensons à la relation de travail, à la théorie des organisations, aux biens collectifs, aux externalités, etc.), y compris pour que l'action soit efficace? L'agent économique

\footnotetext{
${ }^{18}$ Le dernier stade moral, stade 6, caractérisé par la perspective procédurale et l'orientation en fonction de la méthode par justification des normes n'a pas été repris dans ce tableau puisque la confirmation de ce stade n'a pas été établie et qu'il a été introduit à titre hypothétique par Kohlberg et Habermas (Habermas, 1986, pp. 186187).
} 


\section{Une Conception de Moralité pour la Théorie Économique}

théorique peut-il continuer à n’être qu'instrumentalement rationnel - puisque l'agent économique réel, lui, ne l'est manifestement pas?

Nous ne mentionnerons que quelques-unes des critiques faites à Habermas : l'idée d'une rationalité purement procédurale (comme l'est l'éthique de la discussion) a-t-elle un sens? Pourquoi la procédure dialogique conduirait-elle forcément à des décisions justes? Comment une simple forme peut-elle déterminer le fond (puisque Habermas prétend, avec son éthique, répondre à des questions praticomorales du type “que dois-je faire?”) N’y-a-t-il pas déjá un contenu historique implicite dans le choix d'une telle procédure (qui est celle de la science moderne)? Bien évidemment, l'éventuelle pertinence de ces critiques n’enlève rien à la pertinence de la contribution de Habermas.

\subsection{Ricoeur: l'éthique, la morale et la sagesse pratique}

Ricoeur se distingue explicitement de Habermas en ce qu'il ne procède pas par disjonction, par typologie, mais par composition de concepts.

Au concept de morale, Ricoeur (2001, pp. 55-56) assigne une double fonction, celle de désigner,

1. d'une part, la région des normes, c'est-à-dire des principes du permis et du défendu,

2. d'autre part, le sentiment d'obligation en tant que face subjective du rapport d'un sujet à des normes ${ }^{19}$.

Le premier aspect, le caractère obligatoire des normes, qui indique ce qu'il faut faire ou ce qu'il vaut mieux faire, peut prendre un caractère formel, par exemple lorsque les principes satisfont à un test d'universalisation. Mais Ricoeur fait observer, d'une part, que rien n'exige pour autant de tenir le devoir pour l'ennemi du désir, et, d'autre part, que la forme des maximes n'indique pas comment se forment les propositions d'action qui donnent un contenu à la forme du devoir (p. 57). Le deuxième aspect concerne le rapport du sujet à l'obligation, la liberté pratique du sujet de reconnaitre dans les normes une prétention légitime à règler les conduites. La réunion de ces deux aspects, norme objective et face subjective, forme le concept d'auto-nomie, lequel pose la détermination mutuelle de la norme et du sujet. Mais Ricoeur souligne que cette morale, la morale de l'obligation, ne se suffit pas pour autant à elle-même, il lui faut recourir à l'éthique (p.58).

Le concept d'éthique se dédouble également en 1) une éthique "antérieure" qui se situe en amont des normes et qui cherche l'enracinement de celles-ci dans la vie et le désir, et 2) une éthique postérieure

\footnotetext{
${ }^{19}$ Nous retrouvons chez cet auteur une importante place faite aux sentiments moraux, chers à Smith,
} 


\section{Une Conception de Moralité pour la Théorie Économique}

qui se situe en aval et qui vise à insérer les normes dans des situations concrètes. Au premier sens l'éthique désigne une métamorale tandis qu'au second, elle désigne la sagesse pratique, des dispositifs éthiques comme l'éthique médicale, juridique, des affaires, etc. (p.56).

Pour expliquer la nécessité du recours de la morale à l'éthique, Ricoeur part de l'aspect subjectif, du sentiment d'être obligé car “celui-ci marque le point de suture entre le royaume des normes et la vie, le désir” (p.58). Comme il a été dit plus haut, le formalisme ne condamne pas le désir, mais il le neutralise, laissant la question de la motivation sans réponse ${ }^{20}$. Or, ce qui va motiver un sujet à vouloir faire son devoir, ce sont les sentiments moraux. Parmi ces sentiments, Ricoeur élit celui de l'indignation, qui vise en négatif la dignité d'autrui aussi bien que la sienne propre. C'est le refus d'humilier qui fait la différence entre un sujet moral et un sujet physique. "L'ordre des sentiments moraux constitue ainsi un vaste domaine affectif irréductible au plaisir et à la douleur” (p.59).

Par ailleurs, Ricoeur défend la thèse de la non-rivalité des éthiques téléologiques et déontologiques dans la mesure où elles appartiennent à deux plans distincts de la philosophie pratique (p.61). Á propos du débat sur la possibilité d'une morale universelle, Ricoeur propose une définition plus générale de la moralité, définie "par le souhait de vivre bien, avec et pour les autres, dans des institutions justes” (p.268). Il souligne le caractére téléologique de cette approche et aussi l'enchevêtrement des dimensions universelle et historique. Si le souhait de vivre bien est un fait universel, la visée du bien passe par des appréciations raisonnées de ce qui caractérise comme bonne ou mauvaise une action, appréciations contextuelles et historiquement déterminées. "L’idée primitive de justice n'est que le déploiement à l'échelle dialogique, communautaire, institutionnelle du souhait de vivre bien” (p.271).

Même si la moralité est définie, non plus par le souhait de vivre bien, mais par les notions d'obligation et de devoir, la thèse de Ricoeur est que l'universel (tel que définit par Kant, Rawls et Habermas) ne peut qu'être un universel formel, c'est-à-dire sans contenu puisque tout contenu pratique procède finalement du désir et donc du désir de bonheur (p.272). Mais, dit-il, on ne peut pas pour autant en rester au niveau éthique du souhait de vivre bien car la société laisse une place immense aux conflits et à la violence. Est alors née la nécessité d’un tiers qui a pris la forme dans nos sociétés des lois et des sanctions qui délimitent le champ du permis et du défendu. On est ainsi contraint à passer d'une éthique de la vie bonne à une morale de l'obligation. 


\section{Une Conception de Moralité pour la Théorie Économique}

Par ailleurs, l'action exige de compléter les principes formels par des règles d'application soucieuses des contextes historico-culturels. Face à la difficulté de hiérarchiser des biens fondamentalement hétérogènes (marchands et non-marchands, santé et éducation, etc), d'établir une priorité entre biens de rang supérieur, Rawls et Habermas ont remplacé l’idée trop conflictuelle de bien par celle, réputée plus irénique, de juste et d'obligatoire, au prix d'une réduction de la morale à une règle de procédure. Mais le moment tragique de la vie morale n’a été que déplacé et reporté sur le point d'articulation entre l'universel formel et le jugement moral en situation (p.211).

Pour Ricoeur, donc, déontologie et téléologie ${ }^{21}$ sont, non pas irréductibles l'une à l'autre, mais irréductiblement liés, tout comme l'universalisme et le contextualisme. Ceux-ci ne s'opposent pas mais relèvent de deux niveaux différents de la moralité, celui de l'obligation présumée universelle et celui de la sagesse pratique qui prend en charge la diversité des héritages culturels. Si la moralité ellemême doit faire face à ces dilemmes, la science économique, face aux choix économiques à faire et à justifier, peut-elle prétendre pouvoir les éviter?

\section{UNE CONCEPTION DE LA MORALITE POUR L'ECONOMIE}

Le moment est venu de récapituler et de systématiser les choix théoriques annoncés au long de ce texte. Il faut d'emblée remarquer que, si notre propos initial partait de la nécessité d'intégrer des éléments déontologiques à la théorisation du comportement économique, l’incursion dans la philosophie morale nous a mené à conclure à la nécessité d’y introduire également des éléments axiologiques.

Les éléments déontologiques peuvent être introduits dans l'analyse à travers la notion de normes, notion d'ailleurs déjà largement présente dans la littérature économique sans que sa "nature" déontologique soit explicitement reconnue et honorée. Notre choix sur ce point est d'adopter la définition proposée par Ogien, pour qui les normes intègrent trois aspects : le descriptif, l'appréciatif et le prescriptif. Elles impliquent par définition l'existence de sanctions formelles ou informelles mais s'investissent également d'un caractère de désirabilité. Les normes ont un aspect public, objectif et

\footnotetext{
${ }^{20}$ On se souviendra du traitement de cet aspect chez Habermas pour qui la question de la motivation était effectivement le point aveugle de sa morale universaliste; la morale au sens strict a besoin de la moralité sociale (laquelle est axiologique, historique) pour être appliquée.

${ }^{21}$ Les arguments de Ricoeur rappellent parfois ceux de Durkheim référés plus haut
} 


\section{Une Conception de Moralité pour la Théorie Économique}

contraignant. Pour Habermas comme pour Ricoeur, elles ont valeur déontologique, c'est-à-dire qu’elles doivent être respectées pour leur valeur intrinsèque.

Pour donner un exemple de l'importance que peut avoir la prise en compte d'éléments déontologiques, considérons le Dilemme du Prisionnier. D’après la théorie du choix rationnel, les agents optent forcément pour la stratégie de défection (celle-ci étant la stratégie dominante). Il est ainsi logiquement impossible pour eux, et d'atteindre leur utilité maximale possible, et d'atteindre l'optimum collectif (qui correspond à la stratégie de coopération de la part des deux joueurs). Mais, selon la perspective kantienne, la stratégie de coopération ne présente aucune inconsistence logique. En effet, aucun joueur ne peut désirer que la maxime de défection soit un principe universel; au contraire, tout joueur ne peut que désirer que la coopération soit érigée en principe de comportement universel. Dans ce cas, la raison, au sens kantien, s'impose aux désirs : il peut être rationnel de ne pas faire ce que l'on désire faire. Comme le souligne Sugden (1991, p. 756), l'adoption d'une perspective déontologique menace les fondations de la théorie des jeux.

Affirmer le caractère déontologique des normes implique par définition que les contraintes morales ne peuvent être réduites au statut d'instrument pour l'atteinte d'une fin. Le caractère exclusivement instrumental de la rationalité économique ne peut pas rendre compte de cette dimension de l'agent humain. Sugden l'a bien compris qui propose d'ouvrir le débat sur les fondations philosophiques de l'économie, lesquelles, selon lui, doivent être recherchées auprès d’une conception kantienne plutôt que humienne de la rationalité (Sugden, 1991, p. 752).

Selon Hume, la raison doit être considérée comme un instrument au service des passions, c'est-à-dire, comme un instrument pour l'atteinte de fins qui ne peuvent pas elles-mêmes être expliquées par la raison. De même, dans la théorie du choix rationnel, les préférences sont données, elles ne sont pas elles-mêmes l'objet d'une appréciation rationnelle, et ce sont ces fins/préférences que l'on cherche à maximiser. Or, ce que Kant vient affirmer, c'est que, parallèlement à la rationalité instrumentale, domaine des impératifs hypothétiques (si l'on veut atteindre $x$, alors il faut faire $y$ ), il y a une autre forme de rationalité, celle qui génère les impératifs catégoriques. Dans ce cas, les individus sont, par exercice de leur raison, les auteurs des principes qui gouvernent leurs actions et non plus des sujets qui cherchent à satisfaire leurs désirs. La plus haute faculté morale est l'autonomie, et celle-ci consiste à se donner à soi-même une loi ou règle impartiale. L'éthique déontologique se propose d'indiquer ce que l'on doit faire, se propose de décider, rationnellement, des fins à poursuivre ce qui, par définition, est hors du champ de la rationalité instrumentale. 


\section{Une Conception de Moralité pour la Théorie Économique}

Quant à la conception de moralité, nous optons pour celle de Ricoeur au détriment de Habermas et de Kant. Ce concept désigne pour Ricoeur, non seulement les normes, royaume du permis et du défendu, mais aussi les sentiments moraux. La face publique et objective des normes s'accompagne d'une face subjective et la réunion de ces deux aspects forme le concept d'auto-nomie; il y a détermination mutuelle entre la norme et le sujet. L'anthropologie sous-jacente à cette conception n'est pas, on en conviendra, l'anthropologie de l'homo oeconomicus.

C’est également auprès de Ricoeur et de Durkheim que l'on a trouvé les voies d'une cohabitation possible entre éthique déontologique et rationalité conséquentialiste. En effet, si ces deux formes d'intuitions morales existent parmi les êtres humains - la morale de type kantienne étant d'ailleurs la plus proche de la morale de sens commun-, comment une science qui se veut descriptive et prédictive comme l'économie peut-elle en évacuer une de ses théorisations? Durkheim comme Ricoeur (voir 2.3. ci-dessus) refusent d'admettre la séparabilité de l’idée de bien de celle de devoir. Le premier souligne l'irréductibilité de la complexité humaine tandis que le deuxième se "résigne" : la violence dans les rapports humains impose de passer de l'éthique téléologique à la morale déontologique. La science économique doit affronter le "Mal" constitutif des affaires humaines. Ce "Mal", désigné parfois d'opportunisme (ou d'incertitude, ou d'information asymétrique) dans la littérature est érigé en régularité comportementale universelle par la théorie, pour ne pas dire en norme comportementale universelle. Et beaucoup d'économiste font semblant de croire que ce "Mal" est contenable par des contrats. Postuler un comportement plus proche du réel, c'est-à-dire quelquefois moral, ne serait-il pas plus fécond théoriquement et moins nocif normativement?

Les valeurs ont été définies comme des énoncés évaluatifs et prescriptifs axiologiques, c’est-à-dire portant sur le bien et le mal. L’idée forte nous semble être la distinction établie par Ricoeur entre le désiré (par moi) et le désirable/valable (pour moi et pour tous les autres). Si les valeurs ont incontestablement un versant subjectif, elles sont également des étalons de mesure qui transcendent les évaluations individuelles. L’intégration de cette notion, telle que définie par Ricoeur, dans l'analyse économique, met en cause la conception exclusivement subjectiviste de la valeur qui est celle du mainstream. Elle oblige à une distinction entre valeurs et intérêts qui est étrangère à l'analyse économique traditionnelle et y introduit une dimension axiologique. Jointe à l'intégration d'éléments déontologiques, elle impose, une fois encore, une entorse à la rationalité économique instrumentale qui ne se prononce que sur les moyens et non sur les fins de l'action humaine. Se trouve alors mis en cause le deuxième aspect de la rationalité distingué dans la première section de ce texte, qui concerne la discussion des fins et, avec lui, la prétention à la neutralité éthique de la théorie économique. 


\section{Une Conception de Moralité pour la Théorie Économique}

Par ailleurs, l'introduction de la notion de valeurs impose d'ouvir la boite de Pandore des économistes qu'est la notion d'intêrêts et de poser la question des motivations. On l'a vu, cette notion constitue un élément de rupture entre les conceptions éthiques de Ricoeur et de Habermas. Pour ce dernier, les motivations restent hors du champ de la morale universaliste telle qu'il l'a définie et ressort du champ de la moralité sociale. Pour Ricoeur, au contraire, la morale ne se suffit pas à elle-même, elle doit nécessairement recourir à l'éthique par le biais des sentiments moraux. Ce sont ces derniers qui, constituant un point de suture entre le règne des normes et celui du désir, vont motiver les agents à vouloir faire leur devoir. On ne peut s'empêcher de noter l'étrange coincidence : la question des motivations est catégoriquement mise hors du champ de l'économie, cette question est liée à celle des sentiments moraux, laquelle fait justement l'objet de l'oeuvre fondamentale du "père” de l'économie.

C'est l'axiomatique de Savage, la formalisation du choix rationnel sous la forme d'un ordonnancement complet des actions qui évacue les motivations hors de l'économie en la remplaçant par un formalisme logique. La fonction d'utilité empêche de dire quoi que ce soit sur la façon comme se forment les préférences, postulées exogènes. L’ordre de préférence peut refléter une éthique rigoureuse ou un égoisme cynique : rationalité et moralité seraient donc parfaitement compatibles ... si les motivations morales étaient de fait de même "nature" que les autres types de motivations. Or, c'est précisément ce point que contestent les partisans de l'éthique déontologique, les tenants des théories objectivistes de la valeur, et la plupart des auteurs cités dans ce texte. Ricoeur (2001, p.59): “L'ordre des sentiments moraux... est irréductible au plaisir et à la douleur”; Durkheim (1986, p. 78): "les sentiments moraux ... [sont] hors de pair parmi les autres désirs humains”. Les motivations morales ne peuvent être dissolues parmi les autres motivations dans une fonction d’utilité. Il faut trouver autre chose.

Quant à la séparation des domaines de l'économique et du moral, séparation sagement décidée par certains économistes de la fin du 19éme siécle, la thèse n’est plus tenable. Il est patent qu'une part de plus en plus importante de l'activité économique relève de ce que Habermas appelle "l'activité communicationnelle" (coordination dans les organisations, fonctionnement des marchés du travail, distribution du revenu, résolution des problèmes de l'environnement et autres biens collectifs, entre autres). Ricoeur parle du "tragique de la vie morale” qui impose d'articuler l'universel formel et le jugement moral en situation. Ce moment tragique n'investit-il pas la plupart des choix économiques? N'est-il pas le domaine par excellence de l'économie? Face à l'importance vitale de certains choix économiques, la science économique pourra-t-elle continuer longtemps à éviter de l'affronter?

Les conséquences théoriques et méthodologiques de l'intégration de la moralité dans l'économie que l'on vient de proposer sont lourdes. L’individualisme méthodologique est mis en échec sur divers 


\section{Une Conception de Moralité pour la Théorie Économique}

plans. Lorsque Habermas conclut que les normes ne peuvent être fondées en raison de manière monologique, mais requiérent la discussion; lorsque Ricoeur introduit l'”orientation vers autrui" dans sa théorie de l'action et qu'il conçoit celle-ci comme gouvernée par des normes, on est tentée d'imaginer une science économique, non plus basée sur les deux piliers de l'individu et du marché, mais sur trois piliers : l’individu, l'Autre et les règles ${ }^{22}$.

L'assomption des dimensions axiologique et déontologique par la science économique implique de renoncer à une science positiviste et universaliste. Combien de temps encore les économistes résisteront-ils à leur désir intime pour se laisser séduire par le désir, mondain, de faire science?

\footnotetext{
${ }^{22}$ Suivant la typologie de Ricoeur dans son article de 1993. On explorera ailleurs la valeur heuristique de cette typologie pour la théorie économique.
} 


\section{REFERENCES BIBLIOGRAPHIQUES}

BEN-NER Avner, PUTTERMAN Louis (1998) : "Values and Institutions in Economics" in BENNER, PUTTERMAN (dir) : "Economics, values and organizations", Cambridge University Press, Cambridge.

BLACKBURN Simon (1996) : “Dicionário de Filosofia”, Gradiva, Lisboa.

CANTO-SPERBER Monique (dir.) (1992) : "Dictionnaire de Philosophie Morale et Politique”, PUF, Paris.

CALDAS José Maria Caldas (2001) : "Escolhas e Instituições - Análise Económica e Simulação Multiagentes”, Celta, Lisboa

CALDAS José Maria Caldas (2002) : “A economia é uma ciência moral”, comunicação ....

DEMEULENAERE Pierre (1998) : "Les ambiguités constitutives du modèle du choix rationnel" in SAINT-SERNIN et a.

DUPUY Jean-Pierre (1992) : "Le sacrifice et l'envie - Le libéralisme aux prises avec la justice sociale”, Calmann-Lévy, Paris.

DUPUY Jean-Pierre (1994) : “Sciences sociales et sciences cognitives”, École Polytechnique, polycopié.

DUPUY Jean-Pierre (1996) : "Rationalité - Éthique et rationalité" in CANTO-SPERBER Monique "Dictionnaire d'éthique et de philosophie morale”, PUF, Paris.

DURKHEIM Émile (1996) : “Sociologie et Philosophie”, PUF, Paris.

EYMARD-DUVERNAY François (2002) : "Pour un programme d'économie institutionnaliste" in Revue Économique, vol. 53, nº 2, mars 2002.

GAUTHIER David (1986) : “Morals by agreement”, Clarendon Press, Oxford.

HABERMAS Jurgen (1986) : “Morale et communication”, Les Éditions du Cerf, Paris.

LORDON Frédéric

MUNCK : : "L’institution sociale de l'esprit”....

OGIEN Ruwen (1992) : “Normes et valeurs” in Canto-Sperber, (1992)

PICAVET Emmanuel (1998) : “Aspects praxéologiques des principes interpersonnels” in SAINTSERNIN et a.

RICOEUR Paul (1993) : “Avant la loi morale, l’éthique”, Encyclopedia Universalis - Les Enjeux I, Paris.

RICOEUR Paul (1995) : “Réflexion faite - Autobiographie intellectuelle”, Éditions Esprit, Paris. 


\section{Une Conception de Moralité pour la Théorie Économique}

RICOEUR Paul (2001) : “Le Juste 2”, Éditions Esprit, Paris

SAINT-SERNIN Bertrand, PICAVET Emmanuel, FILLIEULE Renaud, DEMEULENAERE Pierre (1998) : “Les modèles de l’action”, PUF, Paris.

SKORUPSKI John (1998) : "Morality and Ethics" in Routledge Encyclopedia of Philosophy, Routledge, London and New-York.

SMITH Adam (1999) : “Théorie des Sentiments Moraux”, PUF, Paris.

SUGDEN Robert (1991) : "Rational Choice : a Survey of Contributions from Economics and Philosophy”, in The Economic Journal, nº 101, July 1991.

THOMAS Alan (1998) : "Values” in Routledge Encyclopedia of Philosophy. 